\title{
Drag and Drop Scaffolding System
}

\author{
G.A.P.Gampathi
}

\begin{abstract}
Anew drag and drop scaffolding system was introduced to overcome construction difficulties with the existing jumping scaffolding system. It was found by applying to a building that it is adequate with construction of high-rise buildings. The system also enables fast construction while minimizing labour cost and construction waste.
\end{abstract}

\section{Introduction:}

Scaffolding is a temporary structure, constructed in order to improve the efficiency of construction. It is extremely important in many structural engineering applications. These include the construction of buildings, bridges, statues, etc.. There are many scaffolding systems available in the market today; starting from the simple ladder up to complicated systems like steel pipe and connection system, wooden scaffolding and steel scaffolding with jump mechanism. The selections of these systems depend on the customer requirements, such as ease of fixing, adjustability, adaptability with the construction site, cost and other objectives of contractors while satisfying worker safety.

There are many problems with existing systems which we encounter in cay-to-day work. Some of them are: more labour requirements for handling the system, messy and unclear worksites, more time required to raise from one level to another and difficulties of dismantling.

\section{Existing Scaffolding Systems:}

\subsection{Wooden Scaffolding}

The system consists of main members and strings in such a way that main members are tied with strings laterally and vertically to form the structure of the scaffolding. The main members are natural wooden pieces such as bamboo, attonia etc. while strings vary from synthetic to natural ropes. Though these systems are very environmentally friendly, there are many disadvantages such as more time taken to setup and lack of safety due to low durability and the low loading capacities of materials. These raw materials cannot be reused for another site and availability is very limited in developed areas. Therefore these systems are only restricted to one storey buildings and minor construction today.

\subsection{Metal Scaffolding}

Metal scaffoldings are very popular in the construction industry as well as scaffoldings manufactures. They have more advantages over wooden scaffoldings, such as durability, material reusability, higher loading capacity, less time of setting up and higher safety. The system can be seen in even high-rise buildings and very complex constructions. The common metal scaffolding system consists of steel pipes and nut and bolt arrangements to connect pipes. Though the system can be supported at base level or at first storey level, it is also supported at each storey level to increase construction safety. The main advantage of this system, over the others is that it can cover very complex constructions. But there are some disadvantages like high labour consumption in the setting up period, creating messy working-sites, higher cost of initial production and dismantling difficulties.

\subsection{Jumping Scaffolding System}

The system was designed to overcome difficulties in metal scaffoldings. The main structure of this system consists of rectangular units which can hang on different stories. Each unit consists of 4 to 5 platforms which are fixed to the main frame. One side of the frame is held by at least four brackets. When a project is awarded the total number of units for the project has to be decided according to crane capacity that is to be used along the perimeter of the structure. Once the units are fixed to the structure at first story level, the main scaffolding erection can be considered as finished.

When the work at second story level is started, all the units have to be lifted to that level and fixed with brackets. The major advantages of this system are less labour cost and room for fast

Eng. G.A.P.Gnupathi, AM(IESL), B.Sr. Eng. (Hars) (Perndeniyn), M.Eng. (NUS), Civil Engineer, Department of Civil Engineering, Naval Dorkjant Trincomalee. 
construction. But there are some difficulties in the lifting processes due to positioning brackets during crane lifting.

\section{Drag \& Drop Scaffolding System:}

\subsection{Introduction}

The system is shown in Fig. 1 ; it can be seen from the figure that it consists of 4 platforms with a maximum span of $10 \mathrm{~m}$ and $12 \mathrm{~m}$ in height. It is an indigenous modular system, which needs only a one-time assembly that effectively encloses the constructing and lower levels during construction. The system was designed to follow the perimeter of the building.
This is unique as it means to be dragged up along its posts. Unlike other scaffolding systems, which hang on the construction level, as they are cranelifted, this just requires to be dragged and dropped in any weather condition. This provides excellent working access and facilitates finishing work safely. To shift the system to the next level, simply rail the units up with a crane. With drag and drop, resetting is so simple, easy, safe and quick. It is designed so that it cannot be displaced from the rail brackets during lifting operations. Units are railed up to the next floor level in just less than 2 minutes. With its huge units, flexible configurations, faster resetting time, safer working methods, it gives more saving in setup and dismantling time, manpower turn-around and site maintenance cost.

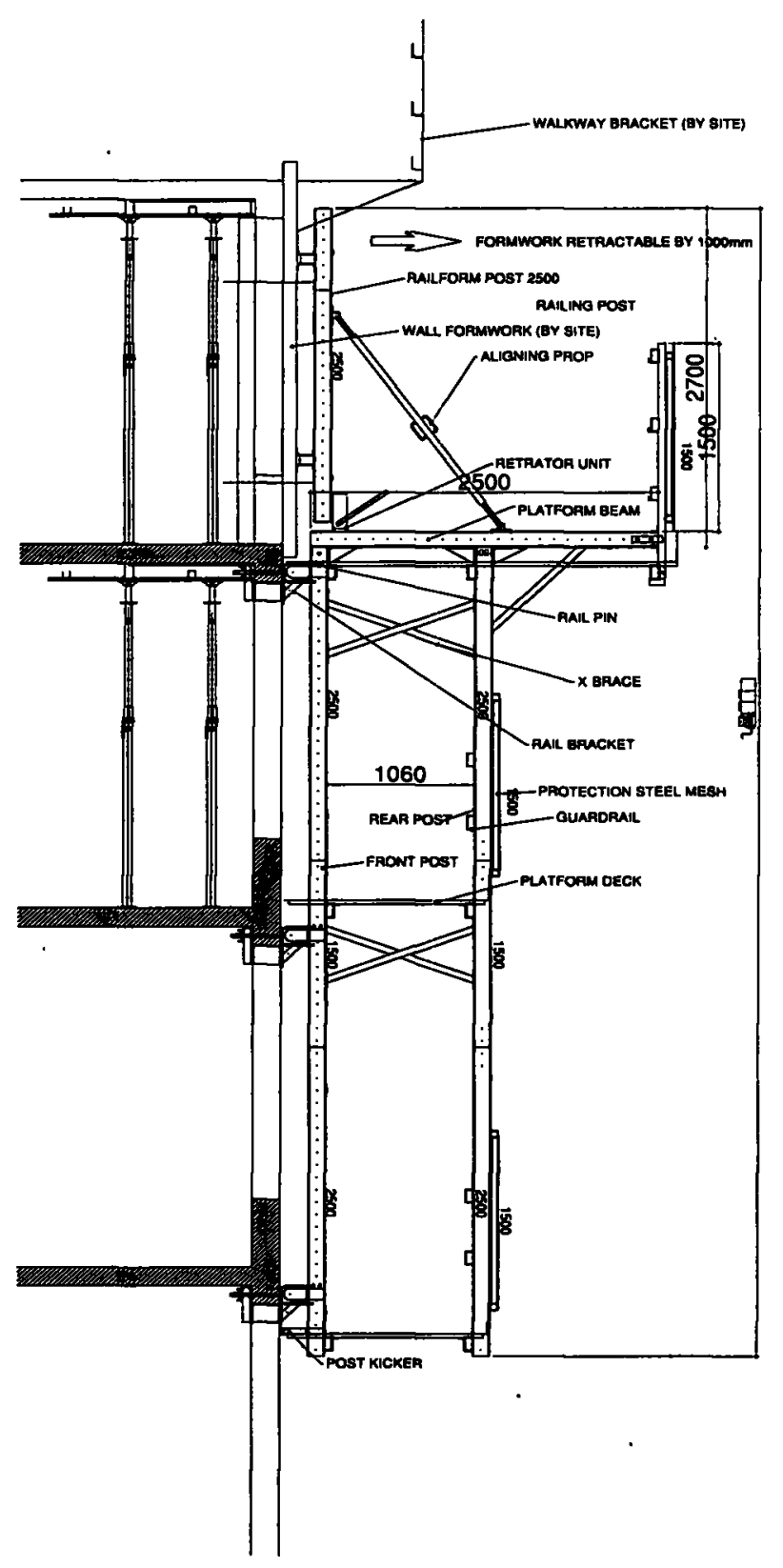

Fig. 1 : Drag and Drop Scaffolding System 


\subsection{Design Specifications:}

- Span $10 \mathrm{~m}$, height $12 \mathrm{~m}$ and with $1.5 \mathrm{~m}$ with platform extension

- All material grade 43 Steel with minimum yield strength $275 \mathrm{~N} / \mathrm{mm}^{2}$

- Dead Load $0.3 \mathrm{KN} / \mathrm{m}^{2}$ on each platform levels + Self-weight

- Live load $2.2 \mathrm{KN} / \mathrm{m}^{2}$ on each platform level

- Wind speed $35 \mathrm{~m} / \mathrm{s}$

\subsection{Details of Brackets and Operational} Procedure

The details of the bracket are shown in Figs. 2.1 and 2.2. It can be seen that two C-channels are welded to a base plate in such a way that opening of the C-channels faces the outside of the assembled brackets. There is a gap between two welded C-channels and a small flat plate is welled in within that gap as shown in the drawing. A tilting arm is assembled to bracket with an M24 bolt that can rotate around the axis of the bolt. But the tilting arm can rotate only 90 degrees since it is stopped on the welded plate between the two C-channels.
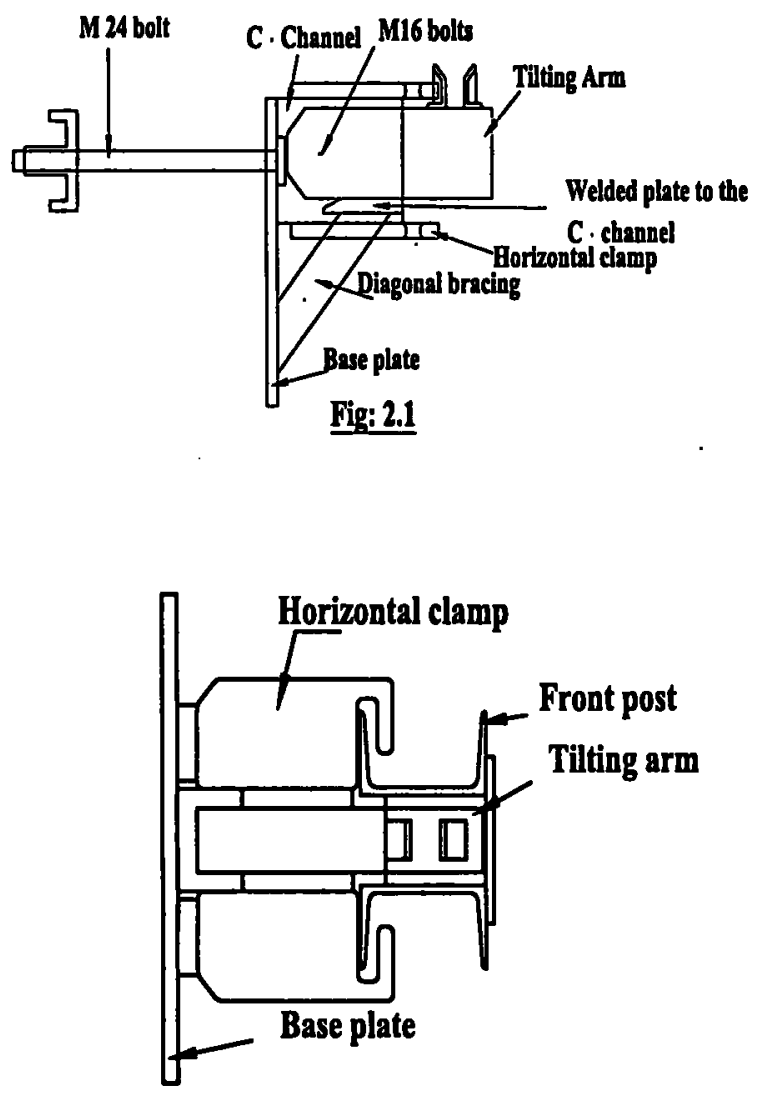

There is a diagonal bracing welded between the plate, which is welded at the gap between Cchannels, and the base plate. This diagonal bracing helps to transfer the vertical load applied on the tilting arm and flat plate to the base plate as a compression force. That is very important in the construction field, since the concrete can withstand much larger compressive forces.

There is a locking/holding mechanism for vertical members that gives a safer system. This locking is introduced with the help of a mechanical component named "horizontal clamp". There are four of these components attached to one bracket in such a way that they can lock the vertical members when required. These components are attached to the bracket with 3 nos. of $16 \mathrm{~mm}$ bolts for each. They are fixed to the C-channels such a way that two of the components are on the topside of the C-channels and the other two are below

\subsection{Operating Mechanism:}

Initially four brackets are fixed to a part of the structure that has already been finished. Then the first unit of the scaffolding system can be fixed to the brackets. After that the construction can be done easily with its help. Once the construction in first story level is finished, the scaffolding unit can be raised easily to the next level. It is necessary to fix two more wall brackets on next level by maintaining the vertical alignment of the bracket lines in order to lift the scaffolding unit by a crane and fix it at the next level. In this way we can raise the scaffolding unit up to the top level of the building or structure easily. This procedure can be used for all the scaffolding units as needed to cover the perimeter of the structure.

\section{Conclusion.}

The new system has many advantages over the other existing systems; it will take a few minutes to lift one unit to the next required level. Since only one time assembly is required at the lower construction level and then it is lifted up for the next, labour requirement is very low. Therefore this system is adequate to reduce labour cost and for fast construction. 